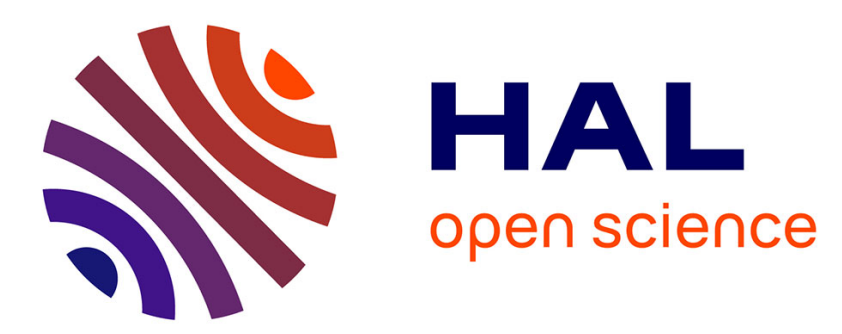

\title{
Adaptive Prognostic of Fuel Cells by Implementing Ensemble Echo State Networks in Time-Varying Model Space
}

\author{
Zhongliang Li, Zhixue Zheng, Rachid Outbib
}

\section{- To cite this version:}

Zhongliang Li, Zhixue Zheng, Rachid Outbib. Adaptive Prognostic of Fuel Cells by Implementing Ensemble Echo State Networks in Time-Varying Model Space. IEEE Transactions on Industrial Electronics, 2019, 67, 10.1109/TIE.2019.2893827 . hal-02475763

\section{HAL Id: hal-02475763 https://hal.science/hal-02475763}

Submitted on 14 Feb 2020

HAL is a multi-disciplinary open access archive for the deposit and dissemination of scientific research documents, whether they are published or not. The documents may come from teaching and research institutions in France or abroad, or from public or private research centers.
L'archive ouverte pluridisciplinaire HAL, est destinée au dépôt et à la diffusion de documents scientifiques de niveau recherche, publiés ou non, émanant des établissements d'enseignement et de recherche français ou étrangers, des laboratoires publics ou privés. 


\title{
Adaptive prognostic of fuel cells by implementing ensemble Echo State Networks in time varying model space
}

\author{
Zhongliang Li, Member IEEE, Zhixue Zheng, Member IEEE, and Rachid Outbib
}

Post Conference Paper

\begin{abstract}
Prognostic plays an important role in improving fuel cells' reliability and durability performance, although it is hard to realize an adaptive prognostic because of the complex degradation mechanisms and the influence of the operating conditions. In this paper, an adaptive datadriven prognostic strategy is proposed for fuel cells operated in different conditions. To extract a feasible health indicator, a series of linear parameter varying (LPV) models are identified in the sliding data segments. Then, virtual steady-state stack voltage is formulated in the identified model space and considered as the health indicator. To enhance the adaptability of prognostic, an ensemble echo state network (ESN) is then implemented given the extracted health indicator data. Long-term tests on a type of low power-scale proton exchange membrane fuel cell (PEMFC) stack in different operating modes are carried out. The performance of the proposed strategy is evaluated using the experimental data.
\end{abstract}

Index Terms-PEMFC, data-driven prognostic, model space, adaptability, health indicator, ESN ensemble.

\section{INTRODUCTION}

W THIN the last two decades, fuel cell (FC) technology has been re-attracting more and more attention on the worldwide scale. Several key criteria, such as FC system overall efficiency and cost control, have been improved significantly and supposed to reach the baseline for wide commercial applications before 2020 [1]. Among different FC types, the polymer electrolyte membrane fuel cell (PEMFC) is one of the most used thanks to its wide power range and flexibility of implementation. Especially, the PEMFC is a promising candidate for power source in various electric vehicles. However, the reliability and durability of current FC technology do not satisfy the requirements for engines in most cases [2].

Among the solutions, the research on Prognostics and Health Management (PHM), dedicated to accessing the future health state and mitigating system failure risks, has been launched besides the ongoing improvements of materials and FC designs. In the PHM framework, the objective of prognostic is to predict the remaining useful life (RUL) before a failure occurs by evaluating the current and historical conditions of a in-service system or a class of similar systems. An effective prognostic can provide the data to carry out condition based maintenance (CBM). The life-cycle costs of the concerned system can therefore be reduced by decreasing inspection, down-time and unscheduled maintenance [3]. Specific to FCs, prognostics can also potentially provide the information to guide the configuration of system operating parameters and to assist in investigating the failure mechanisms.

During the last years, different types of approaches have been proposed for FC prognostics. Among them, model-based approaches can be considered when a physical degradation model is built. The authors of [4] propose to apply extended Kalman filter (EKF) and the inverse first-order reliability method (IFORM) to track the deviations of global resistance and limiting current density, which are considered as the indicators reflecting the state of health. In [5], a physics-based, prognostic-oriented catalyst degradation model is developed and an unscented Kalman filter is applied to predict the trend of the electro-chemical surface area evolution. However, these proposed model-based approaches consider some specific operations and degradation factors. These models may lose their effectiveness when a different FC system design and/or a different operating condition is faced.

The mechanisms of FC degradation are complex. Mechanical, thermal and chemical degradations are involved in different components of a FC stack, such as membrane, gas diffusion layer, bipolar plate [6]. The effects of those degradations are also correlated and highly dependent on the operating conditions [7]. An analytical degradation model with full consideration of all the degradation factors is difficult to build. For this reason, data-driven prognostics and those hybridizing data-driven and model-based principles have become the concerns of numerous studies.

In the framework, several studies based on machine learning techniques or other data-based techniques are proposed to trace the stack voltage evolution. In [8], an adaptive NeuroFuzzy inference approach is proposed to predict the temporal variation of stack voltage. In [9], [10], two alternatives of Neuro Networks (NNs), named extreme learning machine (ELM) and echo state networks (ESN), are applied for the same purpose. An incremental learning of a so-called key performance indicator (KPI) is also proposed to predict long- 
term voltage prediction [11]. A modified relevance vector machine (RVM) is proposed to predict stack voltage evolution in the case of small training datasets [12]. Similarly, Gaussian Process State Space Models (GPSS) are proposed in [13]. In [14], an empirical model for power ageing is proposed. The evolution of the model parameters is tracked by jointing a group of particle filters. To reach a more precise shortterm or medium-term prediction of stack voltage evolution, wavelet analysis, combined with some regression methods is also proposed [15], [16].

These attempts to predict stack voltage are meaningful considering that the research on prognostic methodologies is still in the developing stage. However, these proposals assume that FCs are operated in the constant nominal operating conditions. The deviation of stack voltage in this case is considered to be influenced only by the ageing degradation. When dynamic or time varying operating conditions are applied, it is not appropriate to take only the stack voltage deviation as the health index, because the stack voltage is also influenced by varied operating parameters and system dynamics. Another issue lies in that most of the existing studies do not take the diversity of FC degradations into consideration. It has been revealed that different factors influence $\mathrm{FC}$ degradation in different manners depending on FC system design and operation [7]. To make an adaptive prediction, the prognostic method should be able to generalize in different degradation cases.

This study contributes to FC prognostic by proposing a data-driven strategy. First, a health indicator (HI) formulation and extraction are proposed to take dynamic and time varying operating conditions into consideration, which is developed based on a previous study in [17]. Second, the prognostic adaptability is enhanced by proposing a multi-model structure. Specifically, to extract the proposed HI, linear parameter varying (LPV) model identification is implemented in a series of sliding data segments. Virtual steady-state stack voltage is reconstructed in the identified model space and defined as HI. Following that, multiple ESN models are configured with different parameters and trained using extracted HI data. RUL prediction is then realized by iteratively implementing ensemble ESN models. Long-term experiments are carried out in different operating conditions. The proposed strategy is then verified based on acquired experimental data.

The paper is organized in the following way. In Section III. the concerned FCs and the long-term tests used to validate the proposed approach are presented. Following a general presentation of the strategy in Section III] Section IV]and V]are respectively dedicated to the explanations of health indicator extraction and adaptive prognostic, which serve as the two successive phases of the strategy. Section VI demonstrates the results of the developed strategy on the acquired experimental data. Finally, the study is concluded in Section VII

\section{EXPERIMENTS AND DATA}

A series of long-term experiments are carried out in this study to build the database for FC durability study and to develop PHM methodologies.

\section{A. Fuel cell stacks}

The FC stacks studied here are designed with the structure of an open cathode and a dead-end anode. Some crucial parameters are listed in Table I. A 24 V DC air fan, which functions to supply air and regulate the temperature, is placed on the FC stack.

TABLE I

Paramenters of THE INVESTIGated FC Stack

\begin{tabular}{cc}
\hline FC type & Open cathode/Dead-end anode \\
\hline Active surface & $33.63 \mathrm{~cm}^{2}$ \\
Nominal pressure at hydrogen inlet & $1.35 \mathrm{bar}$ \\
Number of cells & 15 \\
Nominal output power & $73.5 \mathrm{~W}$ \\
Operating temperature & corresponding to current \\
Maximum temperature & $75{ }^{\circ} \mathrm{C}$ \\
Maximum current & $13.45 \mathrm{~A}$ \\
Lowest permitted stack voltage & $7.5 \mathrm{~V}$ \\
Pressure interval at hydrogen inlet & 0.10 to $0.40 \mathrm{bar}$ \\
\hline
\end{tabular}

The references of operating temperatures are provided by FC supplier as a function of the load current:

$$
T_{\text {ref }}=2.5074 I+30.3585
$$

where $T_{\text {ref }}$ denotes the optimal operating temperature, and $I$ is the load current.

The temperature control is realized by regulating the air fan driving voltage. A sufficient quantity of air is guaranteed even in the condition where the air fan is set at the lowest speed. On the anode side, the pressure at the hydrogen inlet is fixed near 1.35 bar. A purge action is activated each $30 \mathrm{~s}$, and each purge lasts $0.5 \mathrm{~s}$. The FCs are self-humidified. No additional humidifier is equipped at the cathode.

\section{B. Database}

Long-term experiments are carried out on different stacks and in different experimental settings, including constant current (CC) mode and dynamic operating mode. During each long-term test, the stack voltage, the individual cell voltages (cell voltages 1 to 15), the temperatures (at the positions of cell 1 , cell 8 , and cell 15), and the stack current are measured with a sample frequency of $5 \mathrm{~Hz}$. Additionally, one polarization curve is collected each $168 \mathrm{~h}$ (one week). Notice that several stops and restarts occur during the long-term tests because of the problems within the test bench, such as the replacement of the hydrogen tank, the problem of central air conditioner of the test room.

In the $\mathrm{CC}$ mode test, the output current was set at $8 \mathrm{~A}$, which is the nominal value. The stack voltage measured during the test is illustrated in Fig. 1. Four stops/restarts during the test are marked in the figure.

In the dynamic mode test, the output current was set according to the real application requirements thanks to the programmable electronic DC load. In practice, the concerned FC is installed in a battery driven light mobile vehicle and functions as a range extender. The FC is enabled when the battery state-of-charge is lower than a predefined threshold. When the FC is enabled, the current increases from 0 to $8 \mathrm{~A}$ 


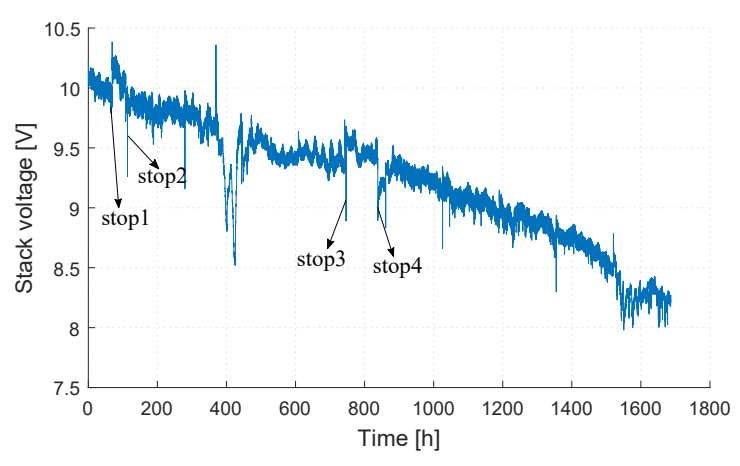

Fig. 1. Stack voltage evolution in CC experimental setting

gradually. The current data during several hours are shown in Fig. 2 The stack voltage during the long-term dynamic test is illustrated in Fig. 3. Three stops/restarts during the test are marked in the figure.

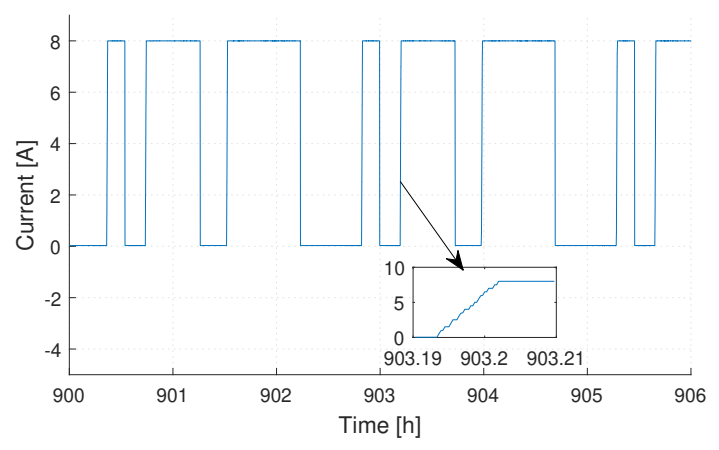

Fig. 2. Current profile in the concerned application

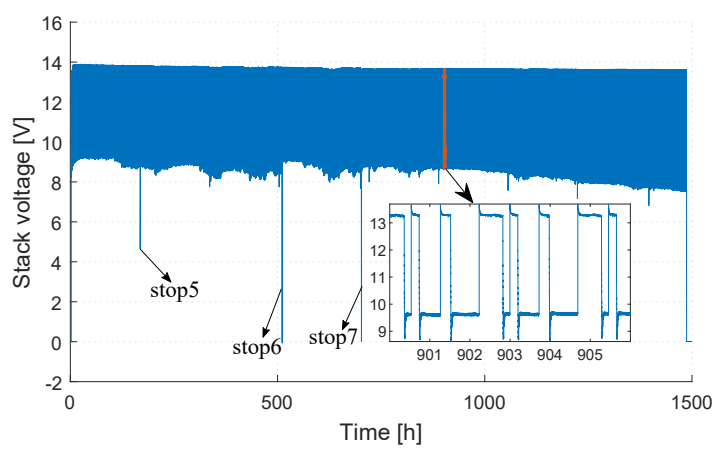

Fig. 3. Stack voltage evolution in dynamic experimental setting

\section{Principle of the prognostic strategy}

In dynamic operating conditions, single FC stack voltage does not show good monotonic trend, which makes it hard to make accurate prediction. Besides, to quantify the RUL, it is also hard to define a feasible failure threshold based on stack voltage in dynamic conditions. For these reasons, this study proposes a two-step prognostic strategy to realize adaptive RUL prediction even in dynamic conditions. As shown in Fig. 4 the prognostic strategy consists of health indicator extraction and ensemble ESN prognostic. In the first step, raw measured input and output data are gathered in sliding segments. In each data segment, the parameters of a LPV model are identified to fit the input and output data. Then, the HI is extracted in the model space, constructed with the identified model parameters. In the second step, fed with the extracted HI data, multiple ESN models with different initializations are trained. The RUL and its confidence interval (CI) can then be estimated by synthesizing the trained ensemble ESN models.

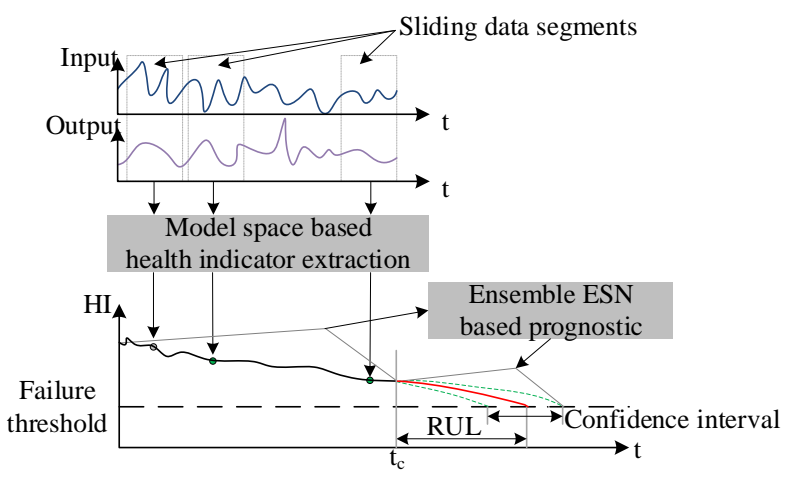

Fig. 4. Illustration of the prognostic strategy

\section{HEALTH INDICATOR EXTRACTION IN MODEL SPACE}

Inherently, the $\mathrm{FC}$ degradation is linked to the variation of FC internal characteristics, which could be quantified generally with system model parameters. Hence, the HI extraction can be realized by learning in model space, i.e., model parameters. In this section, the FC dynamic behavior will be revealed in the LPV model structure. The identification of the model parameters subject to one data segment is presented. Following that, the virtual steady state output, which is formulated in the model space, is defined as the HI and fed to the following prognostic step.

\section{A. LPV-ARX model identification}

In our study, the length of time segment should be set to cover the system dynamics. It should also be much shorter than the ageing degradation cycle. It is assumed that the behavior of a FC system does not vary within a time segment and can be represented by a fixed model. In the concerned data segment, the LPV model is selected here to describe the nonlinear and time-varying properties of PEMFC systems. In [18], LPV model has been demonstrated to be suitable for modeling a commercial PEMFC system. One of the most basic model structures in the LPV context is the so-called auto-regressive model with exogenous input (ARX) [19]. The model is expressed as

$y(k)+\sum_{i=1}^{n_{a}} a_{i}(p(k)) y(k-i)=\sum_{j=0}^{n_{b}} b_{j}(p(k)) u(k-j)+\operatorname{bias}(p(k))+e(k)$

where $k \in \mathbb{Z}$ is discrete time, $u$ and $y$ denote the input and output signals, $p$ is the so-called scheduling variable, bias is 
the system bias which is also dependent on $p, e$ is the system noise. The coefficients $a_{i}$ and $b_{j}$ depend on the instantaneous value of $p(k)$. In this model, the number of time-varying coefficients is $n_{g}=n_{a}+n_{b}+2$. Specific to FC systems, the time-varying property of this model lies on two aspects. First, the system dynamics are dependent on operational points and on the changes among different operational points. The parameters $a_{i}(p(k)), b_{j}(p(k))$ and bias $(p(k))$ can be considered as the functions of the current which is varying with time. Second, the functional dependencies of these parameters are also time varying because of the ageing degradation.

Here, the identification of model parameters is realized by formulating the problem in the least square support vector machine (LS-SVM) form [20]. Without loss of generality, the parameters $a_{i}(p(k)), b_{j}(p(k))$ and bias $(p(k))$ can be reconstructed in the high-dimensional nonlinear functional space of $p(k)$, as

$$
\left\{\begin{array}{l}
a_{i}(p(k))=\omega_{i}^{T} \phi_{i}(p(k)) \\
b_{j}(p(k))=\omega_{\tilde{j}}^{T} \phi_{\tilde{j}}(p(k)), \tilde{j}=j+n_{a}+1 \\
\operatorname{bias}(p(k))=\omega_{n_{g}}^{T} \phi_{n_{g}}(p(k))
\end{array}\right.
$$

where $\phi_{i}: \mathbb{R} \rightarrow \mathbb{R}^{H}$ is a nonlinear function, $\omega_{i} \in \mathbb{R}^{H}$ is the vector in the $H$-dimensional function space. The model in (2) is rewritten in the following form

$$
y(k)=\sum_{i=1}^{n_{g}} \omega_{i}^{T} \phi_{i}(p(k)) x_{i}(k)+e(k)
$$

where

$$
\left\{\begin{array}{l}
x_{i}(k)=y(k-i), \quad i=1, \ldots, n_{a} \\
x_{i}(k)=u\left(k-i+n_{a}+1\right), \quad i=n_{a}+1, \ldots, n_{a}+n_{b}+1 \\
x_{n_{g}}(k)=1
\end{array}\right.
$$

Denoting $\varphi_{i}(k)=\phi_{i}(p(k)) x_{i}(k)$, the problem is further written as

$$
y(k)=\sum_{i=1}^{n_{g}} \omega_{i}^{T} \varphi_{i}(k)+e(k)
$$

which is a regression formulation on $\omega_{i}$. Given $N_{c}$ measured samples, denoted as $\{u(k), y(k), p(k) \mid k=1, \ldots, N\}$, the problem is to find the optimum of $\omega_{i}$ to minimize $e(k)$. This form is similar to the LS-SVM regression formulation. By introducing the regression regularization parameter $\gamma$ and Lagrange multipliers $\boldsymbol{\alpha}=\left[\alpha_{1}, \ldots, \alpha_{N}\right]^{T}$, it can be obtained that [21]

$$
\omega_{i}=\sum_{k=1}^{N} \alpha_{k} \varphi_{i}(k)
$$

The problem (6) can be converted to a problem on $\alpha$, as

$$
y(k)=\sum_{j=1}^{N} \alpha_{j} \sum_{i=1}^{n_{g}} \varphi_{i}(j)^{T} \varphi_{i}(k)+\gamma^{-1} \alpha_{k}
$$

Given $N_{c}$ training samples, this equation can further be written in the vector form

$$
\boldsymbol{Y}=\left(\boldsymbol{\Phi}+\gamma^{-1} \boldsymbol{I}\right) \boldsymbol{\alpha}
$$

where $\boldsymbol{Y}=\left[y(1), \ldots, y\left(N_{c}\right)\right]^{T} ; \boldsymbol{I} \in \mathbb{R}^{N_{c} \times N_{c}}$ is a unit matrix; $\boldsymbol{\Phi}$ is a positive matrix, whose element in the $j$ th row and the $k$ th column is $\Phi_{j k}$ can be expressed as

$$
\Phi_{j k}=\sum_{i=1}^{n_{g}} \varphi_{i}(j)^{T} \varphi_{i}(k)=\sum_{i=1}^{n_{g}} x_{i}(j) \phi_{i}(p(j))^{T} \phi_{i}(p(k)) x_{i}(k)
$$

As the key step of the SVM method, the internal product of function $\phi$ can be expressed as the element of a Kernel matrix, as

$$
K_{j k}^{i}=\phi_{i}(p(j))^{T} \phi_{i}(p(k))
$$

The Radial Basis Function (RBF) kernel used here is defined as:

$$
K_{j k}^{i}=K^{i}(p(j), p(k))=\exp \left(-\frac{\|p(j)-p(k)\|^{2}}{\sigma_{i}^{2}}\right)
$$

Using the kernel function, $\boldsymbol{\Phi}$ can be parameterized, and $\boldsymbol{\alpha}$ in equation (9) can be calculated, as

$$
\boldsymbol{\alpha}=\left(\boldsymbol{\Phi}+\gamma^{-1} \boldsymbol{I}\right)^{-1} \boldsymbol{Y}
$$

Combining (7), the time varying parameters in (3) can be expressed as

$$
\left\{\begin{array}{l}
a_{i}(p(t))=\omega_{i}^{T} \phi_{i}(p(t))=\sum_{k=1}^{N_{c}} \alpha_{k} x_{i} K^{i}(p(k), p(t)) \\
b_{j}(p(t))=\omega_{\tilde{j}}^{T} \phi_{\tilde{j}}(p(t))=\sum_{k=1}^{N_{c}} \alpha_{k} x_{\tilde{j}} K^{\tilde{j}}(p(k), p(t)) \\
\operatorname{bias}(p(t))=\omega_{n_{g}}^{T} \phi_{n_{g}}(p(t))=\sum_{k=1}^{N_{c}} \alpha_{k} x_{n_{g}} K^{n_{g}}(p(k), p(t))
\end{array}\right.
$$

In the FC system studied, the measurable operating parameters are the current and stack temperature. The current is considered as the only input here since the stack temperature is regulated according to the current value. The dynamics involved in the temperature control and FCs can be modeled together. The current is also considered as the scheduling variable. The stack voltage is considered as the output variable.

\section{B. Health indicator extraction}

In the literature, FC stack voltage in nominal operating condition is usually considered as the HI when implementing prognostic. However, this parameter is usually hard to obtain in the dynamic operating conditions. In this study, the virtual steady state output is reconstructed thanks to the identified LPV-ARX model parameters. The extracted virtual steady state output is thus considered as the HI in dynamic conditions.

In the virtual steady state, input, output and scheduling variables are assumed to be constant. In the model of (2), the delayed inputs, outputs and scheduling variables all converge to the steady values, so that

$$
\left\{\begin{array}{l}
y(k-i)=y_{\text {inf }}, i=0, \ldots, n_{a} \\
u(k-j)=u_{\text {inf }}, j=0, \ldots, n_{b} \\
p(k)=p_{\text {inf }}
\end{array}\right.
$$


where $u_{\text {inf }}, p_{i n f}$, and output $y_{\text {inf }}$ are respectively the input, scheduling variable and output in steady state. Ignoring the model error $e(k), 2$ can be transformed into

$$
y_{i n f}+\sum_{i=1}^{n_{a}} a_{i}\left(p_{i n f}\right) y_{i n f}=\sum_{j=0}^{n_{b}} b_{j}\left(p_{i n f}\right) u_{i n f}+\operatorname{bias}\left(p_{i n f}\right)
$$

Thus, the virtual steady state output can be deduced given the input and scheduling variables, as

$$
y_{i n f}=\frac{\sum_{j=0}^{n_{b}} b_{j}\left(p_{i n f}\right) u_{i n f}+\operatorname{bias}\left(p_{i n f}\right)}{\sum_{i=1}^{n_{a}} a_{i}\left(p_{i n f}\right)+1}
$$

The extracted $y_{\text {inf }}$ can thus be considered as the HI and the feature dedicated to prognosis. Notice that multiple features can be extracted by setting different input and scheduling values. The whole HI extraction procedure is summarized in Algorithm 1

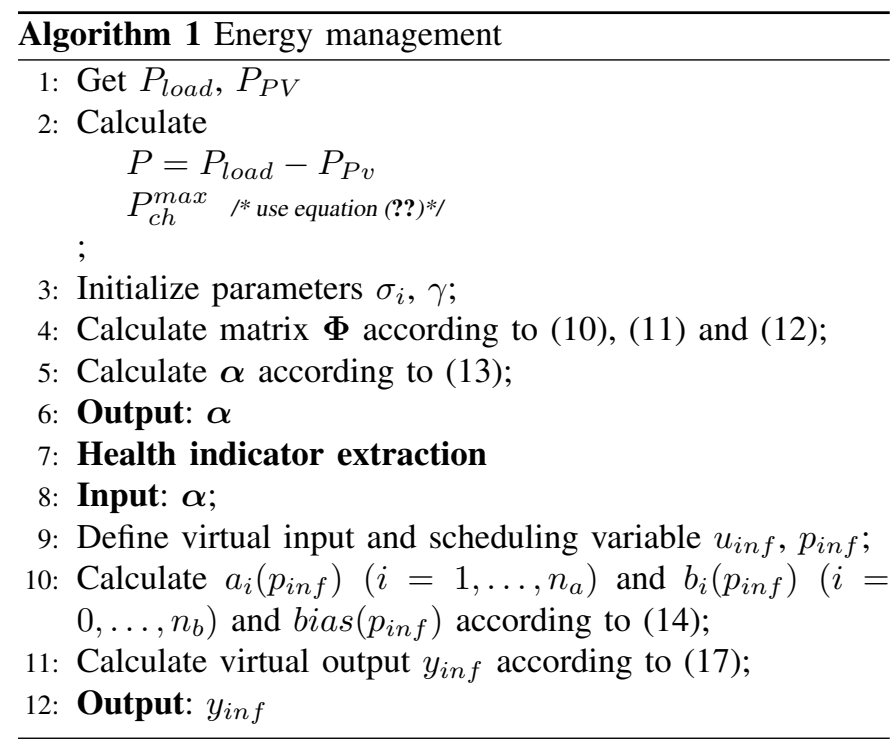

\section{AdAPTIVE PROGNOSTIC BY ENSEMBLE ESNS}

Once the HI is extracted and formed as the training database, a prognostic problem based on the $\mathrm{HI}$ is to be handled. In the data-driven prognostic framework, an ensemble ESN prognostic structure is proposed in this study to obtain an adaptive RUL prediction and the CI.

\section{A. ESN based multi-step prediction}

Recurrent neuron networks (RNNs) have been demonstrated to be very powerful tools for solving temporal learning tasks [22]. ESN, known as an alternative trend of RNNs, has been attracting increasing attention and adapted for prognostics [10]. ESN consists of a non-trainable recurrent part (reservoir) and a simple linear readout. Compared with conventional RNN structures, two advantages make ESN more suitable for long term prediction problems. First, ESN can be trained extremely fast without local optimum. Second, conventional RNNs suffer from the vanishing gradient problem, which makes the memory of RNNs focused only on short term properties of data series [23]. On the contrary, longer term dependencies can be maintained in the ESN structure by setting a sufficiently large neuron number.

The typical update model of ESN is

$$
\begin{aligned}
& \tilde{\boldsymbol{x}}(k)=\tanh \left(\boldsymbol{W}^{i n}[1 ; \boldsymbol{u}(k)]+\boldsymbol{W} \boldsymbol{x}(k-1)\right) \\
& \boldsymbol{x}(k)=(1-\alpha) \boldsymbol{x}(k-1)+\alpha \tilde{\boldsymbol{x}}(k)
\end{aligned}
$$

where $\boldsymbol{x}(k) \in \mathbb{R}^{N_{x}}$ is a vector of reservoir neuron activations and $\tilde{\boldsymbol{x}}(k) \in \mathbb{R}^{N_{x}}$ is its update, at time step $k$. $\boldsymbol{W}^{i n} \in$ $\mathbb{R}^{N_{x} \times\left(1+N_{u}\right)}$ and $\boldsymbol{W} \in \mathbb{R}^{N_{x} \times N_{x}}$ are the input and recurrent weight matrices, respectively. $\alpha \in(0,1]$ is the leakage rate.

$\boldsymbol{W}^{i n}$ and $\boldsymbol{W}$ are randomly generated, subject to the socalled "echo state property": $\boldsymbol{x}(k)$ should be uniquely defined by the fading history of the input $\boldsymbol{u}(k)$ [24].

The output is defined as the linear combination of current states, inputs and an offset term, as

$$
\boldsymbol{y}(k)=\boldsymbol{W}^{\text {out }}[1 ; \boldsymbol{u}(k) ; \boldsymbol{x}(k)]
$$

where $\boldsymbol{y}(k) \in \mathbb{R}^{N_{y}}$ is the network output, $\boldsymbol{W}^{\text {out }} \in$ $\mathbb{R}^{N_{y} \times\left(1+N_{u}+N_{x}\right)}$ is the output weight matrix.

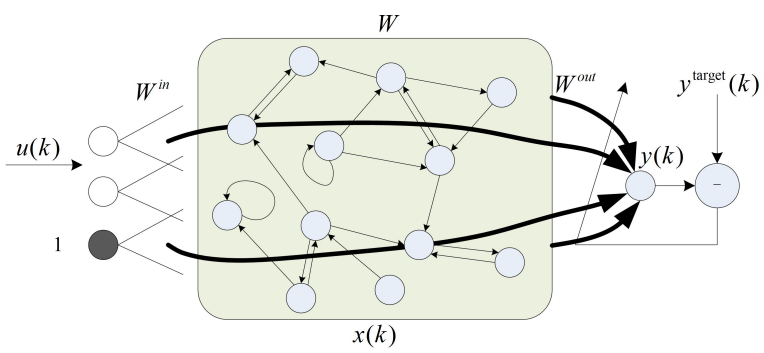

Fig. 5. Illustration of the basic ESN structure, adapted from [24].

The classical ESN is illustrated in Fig. 5. In this structure, only $\boldsymbol{W}^{\text {out }}$ needs to be trained based on the training dataset. When the training dataset is provided, denoted as $\boldsymbol{U}_{t}=$ $\left[\boldsymbol{u}(1), \ldots, \boldsymbol{u}\left(N_{t}\right)\right], \boldsymbol{Y}_{t}=\left[\boldsymbol{y}(1), \ldots, \boldsymbol{y}\left(N_{t}\right)\right]$, the corresponding reservoir states $\boldsymbol{X}_{t}=\left[\boldsymbol{x}(1), \ldots, \boldsymbol{x}\left(N_{t}\right)\right]$ can be calculated according to (18).

The output weight matrix can be realized by ridge regression, as

$$
\boldsymbol{W}^{\text {out }}=\left(\boldsymbol{\Psi}_{t}^{T} \boldsymbol{\Psi}_{t}+\lambda \boldsymbol{I}\right)^{-1} \boldsymbol{\Psi}_{t}^{T} \boldsymbol{Y}_{t}
$$

where $\boldsymbol{I}$ is $N_{x}$ order unit matrix; $\lambda$ is the regulation parameter;

$$
\boldsymbol{\Psi}=\left[\mathbf{1} ; \boldsymbol{U}_{t} ; \boldsymbol{X}_{t}\right]=\left[\begin{array}{cccc}
1 & 1 & \ldots & 1 \\
\boldsymbol{u}(1) & \boldsymbol{u}(2) & \ldots & \boldsymbol{u}\left(N_{t}\right) \\
\boldsymbol{x}(1) & \boldsymbol{x}(2) & \ldots & \boldsymbol{x}\left(N_{t}\right)
\end{array}\right]
$$

In this study, the prognostic on FCs would require modeling the trend of $\mathrm{HI}$ as a function of the time. The input-output of ESN is organized as follows: the input $\boldsymbol{u}(k)$ is composed by the accumulated running time of the FC stack $t(k)$ and the HI of the last two time instances, as

$$
\boldsymbol{u}(k)=\left[t(k) ; y_{\text {inf }}(k-2) ; y_{\text {inf }}(k-1)\right]
$$

while the output is the HI of the current instant, as $\boldsymbol{y}(k)=$ $y_{\text {inf }}(k)$.

Based on a trained ESN model, multi-step prediction can be realized using an iterative one-step prediction method. At the moment $t(k)$, the predicted HI value after $H$ time steps $\hat{y}_{\text {inf }}(k+H)$ can be obtained by $H$ iterations. The iterative realization is illustrated in Fig. 6. 


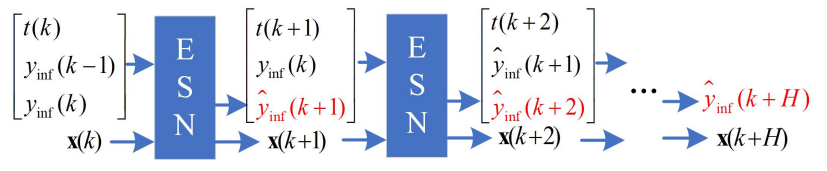

Fig. 6. Illustration of iterative realization of multi-step prediction

\section{B. Ensemble ESN prognostic}

The degradation of FCs is influenced by various phenomena with different time scales and behaviors. Some of these factors may play a more important role on ageing in a specific period. In order to realize an adaptive prognostic, the prognostic model should be capable of modeling this multi-time-scale degradation mechanism. To do this, multiple prognostic ESN models are created and the prognostic is realized by synthesizing the ensemble ESN models. Since multiple models need to be trained in one prognostic cycle, the advantage of the fast training of the ESN models is amplified.

Two crucial parameters, i.e., spectral radius and leakage rate, are concerned to realize a multi-ESN setting. The spectral radius of the reservoir connection matrix $\boldsymbol{W}$, denoted as $\rho(\boldsymbol{W})$, is the maximal absolute eigenvalue of $\boldsymbol{W}$. It determines how fast the influence of an input dies out in a reservoir with time. When $\rho$ is set greater, an extensive history of the input has a more evident influence on the output. The value of leakage rate $\alpha$ highly influences the model dynamics. A smaller $\alpha$ induces slower dynamics of $\boldsymbol{x}(k)$. The two parameters can respectively determine the influence of historical input and the model state updating. It is therefore convincing to initialize these two parameters with different values in order to cover different degradation trends and obtain an adaptive prognostic.

As shown in Fig. 7. $M$ ESN models are initialized with different parameters, denoted as $\left\{E S N_{i}\left(\rho_{i}, \alpha_{i}\right) \mid i=1, \ldots, M\right\}$. With the historical data, these ESN models are trained in the offline phase. In the online phase, multi-step prediction is performed, based on each trained ESN. The mean and the standard variance of the predicted $\mathrm{HI}$ at $k+H$ are calculated as

$$
\begin{gathered}
\bar{y}_{i n f}(k+H)=\frac{1}{M} \sum_{i=1}^{M} \hat{y}_{i n f, i}(k+H) \\
\sigma(k+H)=\sqrt{\frac{1}{M-1} \sum_{i=1}^{M}\left(\hat{y}_{i n f, i}(k+H)-\bar{y}_{i n f}(k+H)\right)^{2}}
\end{gathered}
$$

Assuming that the predictions of multiple ESN models follows standard Gaussian distribution, the CI of prediction can be calculated as

$$
\begin{array}{r}
C I(k+H)=\left[\bar{y}_{\text {inf }}(k+H)-z^{*} \sigma(k+H),\right. \\
\\
\left.\bar{y}_{\text {inf }}(k+H)+z^{*} \sigma(k+H)\right]
\end{array}
$$

where $z^{*}$ is the critical value, which is dependent on the confidence level. For instance, corresponding to the confidence level of $95 \%$ and $98 \%$, the critical value is respectively 1.96 and 2.326 .

The RUL is estimated by comparing the mean prediction $\bar{y}_{\text {inf }}(k+H)$ with the predefined failure threshold. The CI of

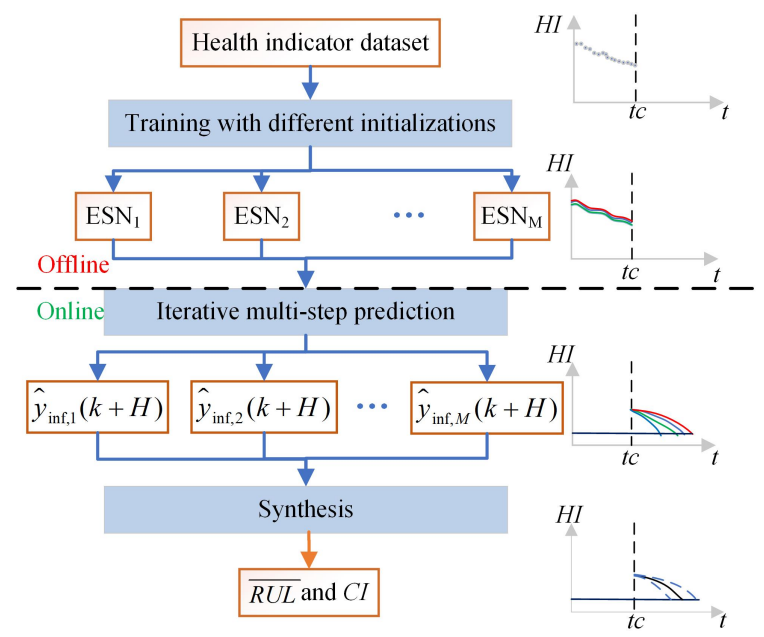

Fig. 7. ESN ensemble prognostic implementation

RUL is calculated by comparing the lower and upper limits of $C I(k+H)$ with the same failure threshold.

\section{Results AND Discussions}

In this section, the effectiveness of the proposed prognostic strategy is demonstrated using the experimental data acquired during the long-term tests presented in Section III

\section{A. Prognostic in constant current operating mode}

In the case of $\mathrm{CC}$ operating condition, the FC stack voltage can be considered as the HI. The health indicator extraction step can thus be skipped. The prognostic based on ensemble ESNs will be implemented directly on the stack voltage data series shown in Fig. 1. After normalizing the data to the interval $[0,1]$, the elements of input matrix $\boldsymbol{W}^{\text {in }}$ are generated randomly and uniformly between -0.5 and 0.5 . The number of recurrent neurons is set to 400 . The recurrent weight matrix $\boldsymbol{W}$ is initialized randomly. The spectral radius $\rho$ of $\boldsymbol{W}$ and the leakage rate $\alpha$ are then parameterized with different parameters, as $\{\rho, \alpha \mid \rho \in[1.0: 0.05: 1.3], \alpha \in[0.1: 0.1: 0.9]\}$. Thus, 63 models are created by setting different parameters. The ranges of these two parameters are set according to the principle provided in [24].

When the first $500 \mathrm{~h}$ data are used to train the ensemble ESN models, the real measured stack voltage and the estimated results from the trained ESN models are shown in Fig. 8 It can be seen that all the ESN models, even initialized with different parameters, can well reflect the input-output relation of the training data. Two sags are observed between $400 \mathrm{~h}$ and $480 \mathrm{~h}$. During this period, the air conditioner installed in the test room failed. Correspondingly, the FC stack was operated with higher temperature. This may introduce more water on the cathode side of the FCs and lower the performance of FCs. These data have also been used for training the ESN models. Thanks to the modeling capability of the ESN, the abnormal degradation behavious can be well modeled.

When the prognostic is implemented by using the multiple trained models for the rest data, the multi-step predictions and 


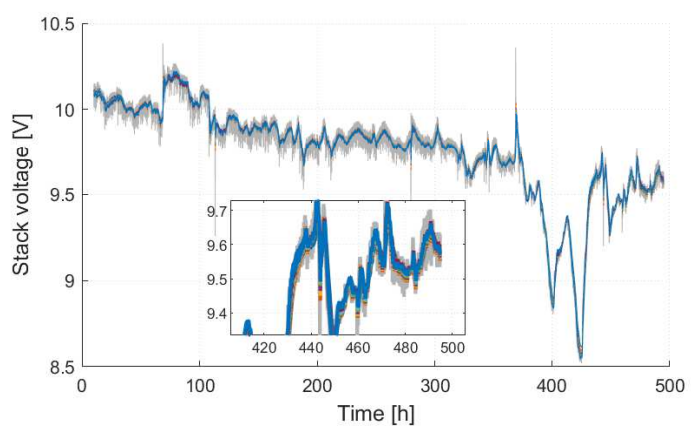

Fig. 8. Training results of ensemble ESN models. Grey line: measured stack voltage evolution; Colorful lines: estimated results from the trained ESN models.

the real measurement are shown in Fig. 9(a), The diversity depending on parameter configurations can be seen. The mean prediction and the CI of $95 \%$ confidence level are illustrated in Fig. 9(b), The mean prediction can follow the degradation trend of the seen data, although the difference between the estimation and the real data increases as the prediction steps accumulate and some unexpected changes occur after the prediction moment. The prognostic can be adapted quickly to the real degradation trend after the unexpected incident between $400 \mathrm{~h}$ and $480 \mathrm{~h}$.

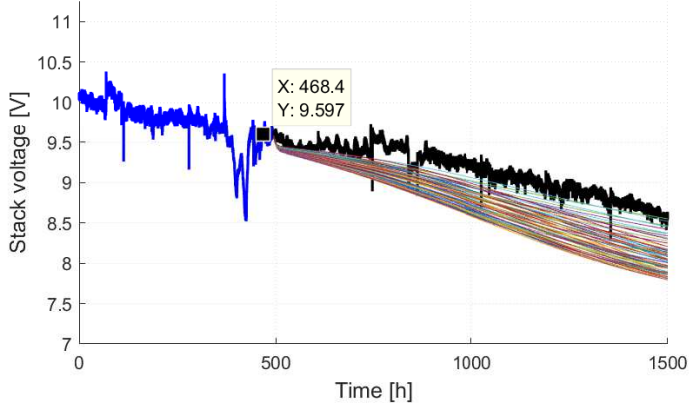

(a)

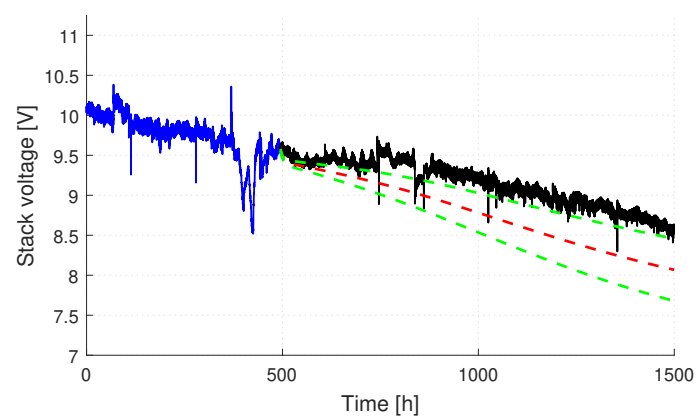

(b)

Fig. 9. Prognostic result by implementing ensemble ESN models with $1000 \mathrm{~h}$ prediction length. Blue line: data used for training ESN ensemble models; Black line: measured voltage evolution. (a) Prediction results of multiple models (colorful thin lines). (b) Mean predicted result (red dotted line) and $\mathrm{Cl}$ (green dotted line).

In this study, the failure threshold is set as the same quantity as the last concerned measurement. The predicted RUL and real RUL at different time points are shown in Fig. 10 The mean of predicted RUL is consistent with the real RUL when the prediction time is close to its end of life. However, some unexpected and irregular changes of the data can introduce incorrect predictions. For instance, the data between $750 \mathrm{~h}$ and $850 \mathrm{~h}$ show a higher level, and the estimated RUL made during this period is therefore higher than the real one. Actually, the irregular changes happening at $750 \mathrm{~h}$ and $850 \mathrm{~h}$ are related to two stop/restart actions, marked as stop3 and stop4 in Fig. 1]. during the test.

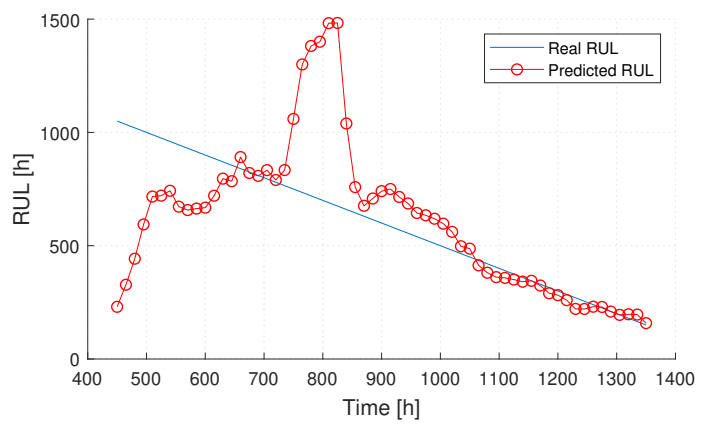

Fig. 10. RUL prediction at different moments in CC operating mode.

\section{B. Prognostic in dynamic operating mode}

In dynamic operation mode, the $\mathrm{HI}$ is firstly extracted in LPV model space. As indicated in Algorithm 11. LPVARX model identification is implemented for a series of data segments which are generated by sliding a fixed-size window within the whole data span. The model identification performance in one data segment is illustrated in Fig. 11 Fig. 11(a), Fig. 11 b) show the current, the stack voltage in one data segment. From Fig. 11 b), it can be seen that the input-output relation can be well described by the proposed LPV-ARX model. Fig. 11. (c) shows the details of the stack voltage in transition. In dynamic processes, the steady-state stack voltage cannot be measured directly.

Efforts are taken here to rebuild the steady-state stack voltage, i.e., the HI, via the identified LPV-ARX model parameters. In the identified model space, $\mathrm{HI}$ is extracted as presented in Section IV-B. The virtual input and scheduling variable, i.e., current, is set at 8 A. The virtual output, i.e., stack voltage, over the whole time span is extracted and shown in Fig. 12. Although the stack voltage is finally reconstructed as the HI, it is not a real stack voltage, but a virtual one which is extracted by considering both operating conditions and system dynamics. Compared with the stack voltage measured in the $\mathrm{CC}$ mode test, the extracted $\mathrm{HI}$ exhibits less monotonous trend. Inherently, the degradation of FCs in dynamic operation is uneven over the lifecycle. The sudden drop of number 3 is linked to the stop caused by replacing the hydrogen tank. Some sags, which are numbered $1,2,4,5$ and 6 in the figure, are observed in the HI data. During these periods, the HI decreases firstly, and recovers to a relatively high value. A compact design and a simple control strategy are adopted for the studied FC system. The 

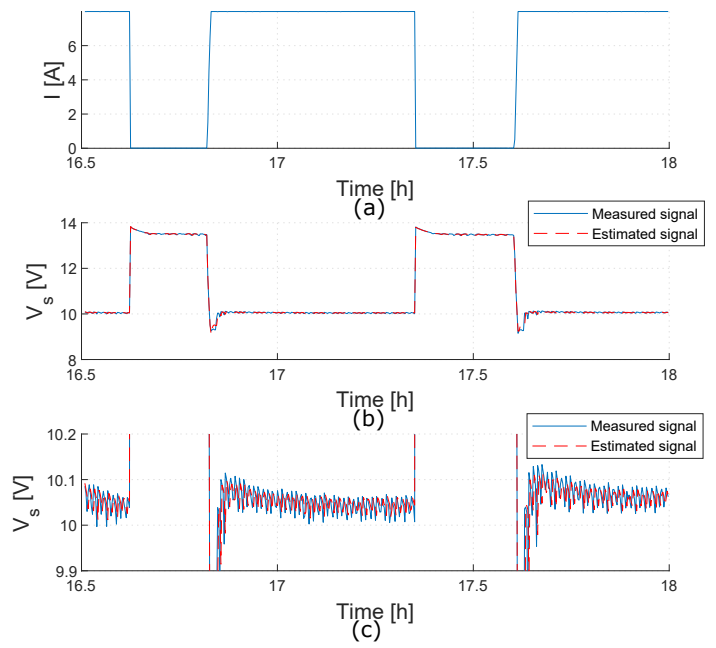

Fig. 11. Model identification performance in one data segment.

flow rates, the temperatures and relative humidities of both air and hydrogen are not controlled. These operating parameters are instable in dynamic conditions, and they are influenced by the test environment. Some recoverable faults or abnormal states could be encountered for the FCs operated in instable and varying conditions. The less monotonous trend of $\mathrm{HI}$ data makes the prognostic harder than in the $\mathrm{CC}$ condition.

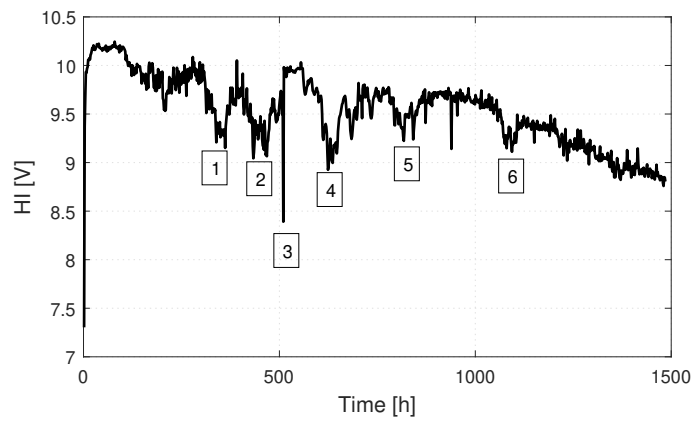

Fig. 12. Extracted $\mathrm{HI}$ in dynamic long-term test.

The prognostic based on ensemble ESNs is implemented on the extracted HI. The multiple ESN models are configured as those in the $\mathrm{CC}$ case. The HI prediction results obtained by implementing the trained multiple ESN models at two moments are shown in Fig. 13 The data acquired before the prediction moment are used for training the ESN models. It can be observed that even though the training data can be fitted well with all ESN models, the predictions by different models show different behaviors. The degradation of the FCs can be influenced by various factors of different time scales. A single ESN model may perform well in one degradation case, but it may also lose its effectiveness in another case. To make the prognostic more adaptive, the multiple prediction results are synthesized as described in the previous section.

The prognostic results and the corresponding CIs obtained by synthesizing ensemble ESN models at 4 different moments are shown in Fig. 14. It can be seen that the real HI measurements are generally enclosed in the CI of $95 \%$ except the case in Fig. 14(c), which means that the diversity of ESN models can generally cover different degradation trends. In the multi-step long-term prediction, the predicted HI means to trace the trend of the past data. It becomes inaccurate when a disturbance occurs. After 750 h, two principle changes can be observed and numbered with 5 and 6 in Fig. 14. In Fig. 14(a) the prediction of RUL is precise because the changes after $750 \mathrm{~h}$ are averaged. The degradation after these two changes just follows the mean prediction of ensemble ESNs, which are trained based on the data before $750 \mathrm{~h}$. In Fig. 14(b) the $\mathrm{HI}$ is recovered after the change 5 and the prediction of degradation becomes more positive. The degradation behavior between $900 \mathrm{~h}$ and $1050 \mathrm{~h}$ shows a monotonous trend. The prediction at $1050 \mathrm{~h}$, shown in Fig. 14(c), generally follows the data before this moment. The unexpected change 6 happens at about $1050 \mathrm{~h}$, and the HI degradation behavior changes following change 6 . Because of this unexpected change, the predictions made at $900 \mathrm{~h}$ and $1050 \mathrm{~h}$ are not consistent with the real HI degradation following change 6. Also, because of this sudden change, the real HI goes out of the CI at 1050, as shown in Fig. 14(c). After change 6, the degradation of the HI becomes more traceable since the data show a more monotonous trend. The prediction becomes more reliable at $1200 \mathrm{~h}$, as shown in Fig. 14(d).

One interesting phenomenon observed is that the ensemble prediction is able to trace the short-term and long-term trend. For instance, in Fig. 14(a) the mean prediction (red dotted line) shows a depression-like behavior at the beginning of the prediction. This special trend can be related to the behavior of the data near the prediction time. After about 150 hours, the predicted degradation becomes monotonously decreasing, which corresponds to the long-term trend. A similar phenomenon is also observed in Fig. 14(b)

By setting the failure threshold the same as the last $\mathrm{HI}$ sample, the predicted RUL and real RUL at different time points are shown in Fig. 15. It is interesting to observe that evolution of the predicted RUL is linked to that of the HI data. Corresponding to the two valleys in changes 5 and 6 , shown in Fig. 14, two underestimations of RUL can be observed at about $820 \mathrm{~h}$ and $1100 \mathrm{~h}$. Between change 5 and 6, the overestimation is observed. The RUL prediction becomes precise after change 6 since the data become more monotonous. The prediction made by ensemble ESN models can quickly adapt the new degradation trend of the data. A reliable prognosis result can be obtained when the prediction horizon is within $350 \mathrm{~h}$.

\section{Discussion}

In this study, the concerned FC stacks are open-cathode and dead-end ones which benefit from a compact design and ease of use. The temperature control and air supply are fulfilled by a single air fan. The pressures, temperatures and relative humidities of both hydrogen and air inlets are not precisely controlled. In other words, the functioning condition of the stack is far from an ideal condition. For this reason, the degradation rates of the concerned stacks are relatively high 


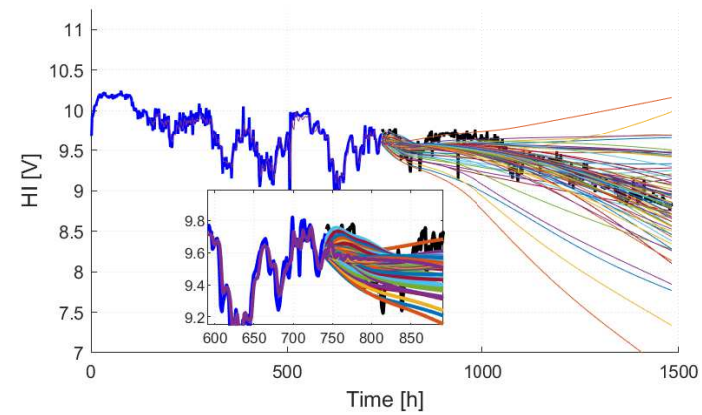

(a)

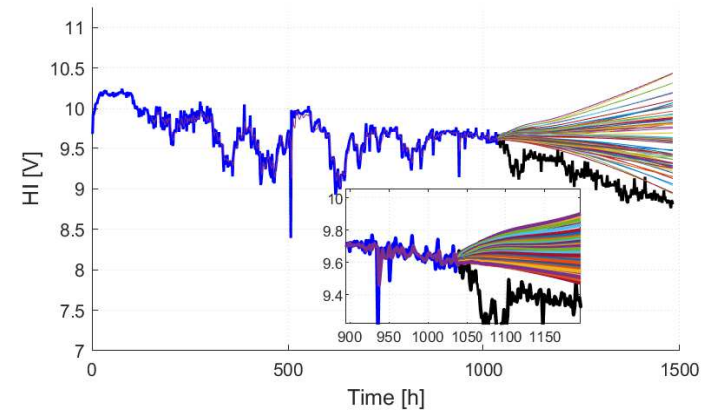

(b)

Fig. 13. Prediction results by implementing multiple ESN models. Blue line: data used for training ESN ensemble models; Black line: measured voltage evolution. Colorful thin lines: predicted results of multiple models. (a) Prediction result at $750 \mathrm{~h}$ (b) Prediction results at $1050 \mathrm{~h}$.

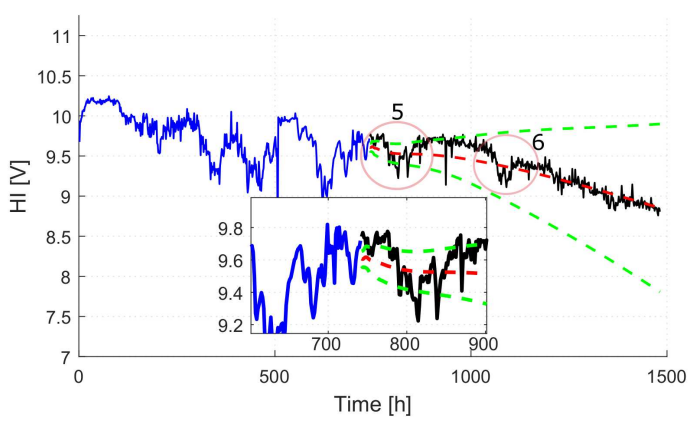

(a)

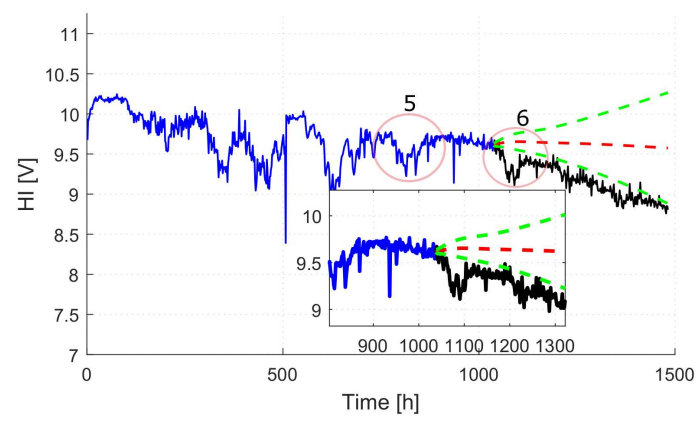

(c)

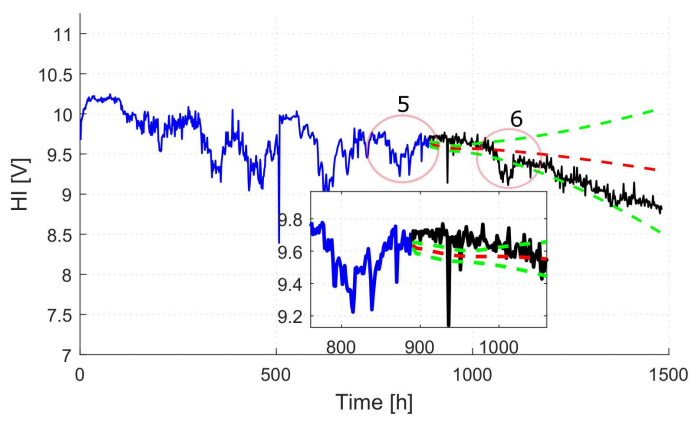

(b)

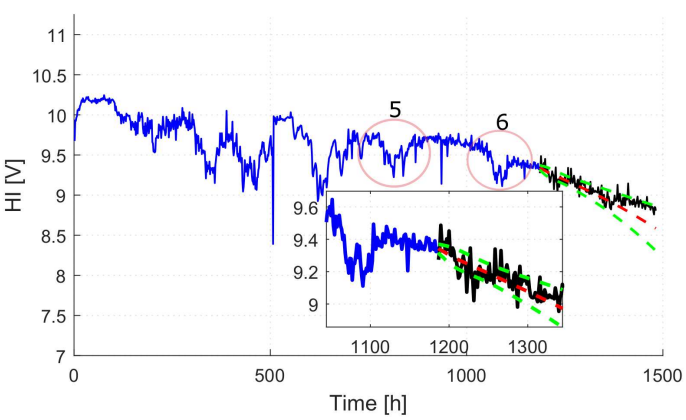

(d)

Fig. 14. Prognostic result by implementing ensemble ESN models. Blue line: data used for training ESN ensemble models; Black line: measured voltage evolution. (a) Mean predicted result (red dotted line) and $\mathrm{Cl}$ (green dotted line) at $750 \mathrm{~h}$ (b) Mean predicted result and Cl at $900 \mathrm{~h} \mathrm{(c)} \mathrm{Mean}$ predicted result and $\mathrm{Cl}$ at $1050 \mathrm{~h}$ (d) Mean predicted result and $\mathrm{Cl}$ at $1200 \mathrm{~h}$.

compared with the records or the data in the literature. It can also be expected that when a better equipped fuel cell system is treated using the same approach, the HI data could be more traceable and the prognostic result with respecting to RUL long-term prediction could be better.

To configure the ensemble ESN models, the separation scales in the parameter ranges and the number of ESN models can be set differently. The final prognostic result, including the estimations of RUL and CI, can be varied with a different set. More detailed work is required for this analysis.

Although multiple ESN models are adopted, the computing time of one prognostic cycle takes less than one minute in 64bit Matlab, 3.4-GHz and 16-G RAM environment, thanks to the fast training of ESN model. The computing time is short enough for estimating the ageing degradation of FCs whose time cycle is often from hundreds to thousands of hours.

\section{CONCLUSIONS}

This paper presents an adaptive data-driven prognostic strategy for FCs. Virtual steady-state stack voltage is formulated as $\mathrm{HI}$ and extracted in LPV model space. The adaptive prediction of HI evolution is realized by implementing ensemble ESN models. Long-term experiments in different operating modes are carried out to validate the proposed approach. From the prognostic results, the following conclusions can be made: 


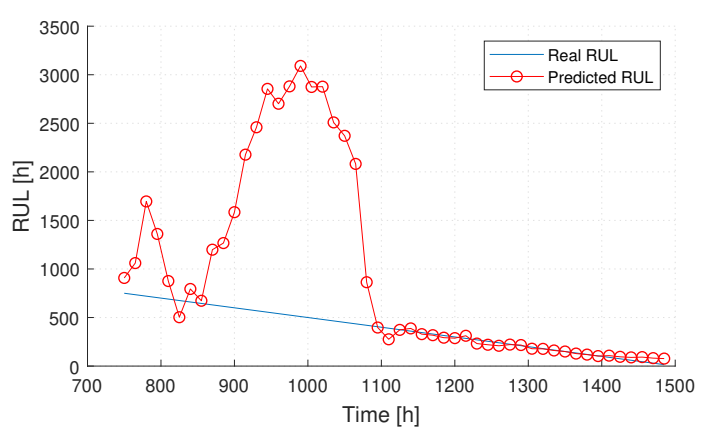

Fig. 15. RUL prediction at different moments in dynamic operating mode.

1) LPV-ARM model is suitable for modeling the studied FC system dynamics. The virtual steady-state stack voltage can be reconstructed in the identified model space and considered as the HI.

2) ESN is validated to be an effective tool for long-term HI prediction. By initializing the ESN model with different parameter combinations, diverse degradation behaviors can be modeled. The prognostic adaptability is thus enhanced.

3) In the case studied, an acceptable prognostic can be achieved when the prediction horizon is within 350 hours in both CC mode and dynamic mode.

In the future work, more long-term tests concerning different FC system designs and controls and different failure modes will be carried out to verify the performance of the proposed strategy. Efforts will also be taken to improve the robustness of the approach to the recoverable disturbances.

\section{REFERENCES}

[1] L. Eudy and M. B. Post, "Fuel cell buses in us transit fleets: Current status 2017," National Renewable Energy Lab.(NREL), Golden, CO (United States), Tech. Rep., 2017.

[2] J. Wang, "System integration, durability and reliability of fuel cells: Challenges and solutions," Applied Energy, vol. 189, pp. 460 - 479, 2017.

[3] N. M. Vichare and M. G. Pecht, "Prognostics and health management of electronics," IEEE Transactions on Components and Packaging Technologies, vol. 29, no. 1, pp. 222-229, March 2006.

[4] M. Bressel, M. Hilairet, D. Hissel, and B. O. Bouamama, "Remaining useful life prediction and uncertainty quantification of proton exchange membrane fuel cell under variable load," IEEE Transactions on Industrial Electronics, vol. 63, no. 4, pp. 2569-2577, April 2016.

[5] X. Zhang and P. Pisu, "An unscented kalman filter based approach for the health-monitoring and prognostics of a polymer electrolyte membrane fuel cell," Annual Conference of the Prognostics and Health Management Society 2012, vol. 3, pp. 1-9, 2012.

[6] J. Wu, X. Z. Yuan, J. J. Martin, H. Wang, J. Zhang, J. Shen, S. Wu, and W. Merida, "A review of pem fuel cell durability: Degradation mechanisms and mitigation strategies," Journal of Power Sources, vol 184, no. 1, pp. 104-119, 2008.

[7] C. Lim, L. Ghassemzadeh, F. Van Hove, M. Lauritzen, J. Kolodziej, G. Wang, S. Holdcroft, and E. Kjeang, "Membrane degradation during combined chemical and mechanical accelerated stress testing of polymer electrolyte fuel cells," Journal of Power Sources, vol. 257, pp. 102-110, 2014.

[8] R. Silva, R. Gouriveau, S. Jemeï, D. Hissel, L. Boulon, K. Agbossou, and N. Y. Steiner, "Proton exchange membrane fuel cell degradation prediction based on adaptive neuro-fuzzy inference systems," International Journal of Hydrogen Energy, vol. 39, no. 21, pp. 11128 - 11144 2014.
[9] K. Javed, R. Gouriveau, N. Zerhouni, and D. Hissel, "Improving accuracy of long-term prognostics of pemfc stack to estimate remaining useful life," in 2015 IEEE International Conference on Industrial Technology (ICIT), March 2015, pp. 1047-1052.

[10] S. Morando, S. Jemei, R. Gouriveau, N. Zerhouni, and D. Hissel, "Fuel cells prognostics using echo state network," in IECON 2013 - 39th Annual Conference of the IEEE Industrial Electronics Society, Nov 2013, pp. 1632-1637.

[11] S. Yin, X. Xie, J. Lam, K. C. Cheung, and H. Gao, "An improved incremental learning approach for kpi prognosis of dynamic fuel cell system," IEEE Transactions on Cybernetics, vol. 46, no. 12, pp. 31353144, Dec 2016.

[12] Y. Wu, E. Breaz, F. Gao, and A. Miraoui, "A Modified Relevance Vector Machine for PEM Fuel-Cell Stack Aging Prediction," IEEE Transactions on Industry Applications, vol. 52, no. 3, pp. 2573-2581, 2016.

[13] L. Zhu and J. Chen, "Prognostics of pem fuel cells based on gaussian process state space models," Energy, vol. 149, pp. 63 - 73, 2018.

[14] M. Jouin, R. Gouriveau, D. Hissel, M. C. Péra, and N. Zerhouni, "Joint particle filters prognostics for proton exchange membrane fuel cell power prediction at constant current solicitation," IEEE Transactions on Reliability, vol. 65, no. 1, pp. 336-349, March 2016.

[15] M. Ibrahim, N. Y. Steiner, S. Jemei, and D. Hissel, "Wavelet-Based Approach for Online Fuel Cell Remaining Useful Lifetime Prediction," IEEE Transactions on Industrial Electronics, vol. 63, no. 8, pp. 50575068, 2016.

[16] H. Liu, J. Chen, M. Hou, Z. Shao, and H. Su, "Data-based short-term prognostics for proton exchange membrane fuel cells," International Journal of Hydrogen Energy, vol. 42, no. 32, pp. 20791 - 20 808, 2017.

[17] Z. Li, S. Jemei, R. Gouriveau, D. Hissel, and N. Zerhouni, "Remaining useful life estimation for pemfc in dynamic operating conditions," in 2016 IEEE Vehicle Power and Propulsion Conference (VPPC), Oct 2016, pp. 1-6.

[18] S. de Lira, V. Puig, J. Quevedo, and A. Husar, "Lpv observer design for pem fuel cell system: Application to fault detection," Journal of Power Sources, vol. 196, no. 9, pp. 4298 - 4305, 2011, cONAPPICE 2010. [Online]. Available: http://www.sciencedirect.com/science/article/ pii/S0378775310020756

[19] A. Golabi, N. Meskin, R. Tóth, and J. Mohammadpour, "A Bayesian Approach for LPV Model Identification and Its Application to Complex Processes," IEEE Transactions on Control Systems Technology, vol. 25, no. 6, pp. 2160-2167, Nov 2017.

[20] Tóth, Roland and Laurain, Vincent and Zheng, Wei Xing and Poolla, Kameshwar, "Model structure learning: A support vector machine approach for LPV linear-regression models," IEEE Conference on Decision and Control and European Control Conference, pp. 3192-3197, dec 2011.

[21] H. Wang and D. Hu, "Comparison of svm and ls-svm for regression," in ICNN\&B'05. International Conference on Neural Networks and Brain, 2005, vol. 1. IEEE, 2005, pp. 279-283.

[22] B. Schrauwen, D. Verstraeten, and J. Van Campenhout, "An overview of reservoir computing: theory, applications and implementations," in Proceedings of the 15th European Symposium on Artificial Neural Networks. p. 471-482 2007, 2007, pp. 471-482.

[23] G. Holzmann and H. Hauser, "Echo state networks with filter neurons and a delay\&sum readout," Neural Networks, vol. 23, no. 2, pp. 244 256, 2010.

[24] M. Lukoševičius, "A practical guide to applying echo state networks," in Neural networks: Tricks of the trade. Springer, 2012, pp. 659-686. 